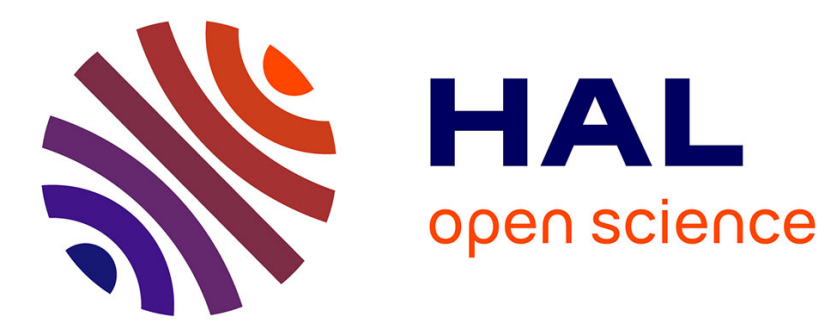

\title{
Electron Rephasing in a Laser-Wakefield Accelerator
} Emilien Guillaume, Andreas Döpp, Cédric Thaury, Agustin Lifschitz, G Grittani, J.-P Goddet, A Tafzi, S W Chou, L Veisz, Victor Malka

\section{To cite this version:}

Emilien Guillaume, Andreas Döpp, Cédric Thaury, Agustin Lifschitz, G Grittani, et al.. Electron Rephasing in a Laser-Wakefield Accelerator. Physical Review Letters, 2015, 115, pp.155002. 10.1103/PhysRevLett.115.155002 . hal-01220095

\section{HAL Id: hal-01220095 https://hal.science/hal-01220095}

Submitted on 27 Oct 2015

HAL is a multi-disciplinary open access archive for the deposit and dissemination of scientific research documents, whether they are published or not. The documents may come from teaching and research institutions in France or abroad, or from public or private research centers.
L'archive ouverte pluridisciplinaire HAL, est destinée au dépôt et à la diffusion de documents scientifiques de niveau recherche, publiés ou non, émanant des établissements d'enseignement et de recherche français ou étrangers, des laboratoires publics ou privés. 


\title{
Electron Rephasing in a Laser-Wakefield Accelerator
}

\author{
E. Guillaume, ${ }^{1}$ A. Döpp, ${ }^{1,2}$ C. Thaury, ${ }^{1}$ K. Ta Phuoc, ${ }^{1}$ A. Lifschitz, ${ }^{1}$ G. Grittani, ${ }^{3,4}$ J.-P. Goddet, ${ }^{1}$ \\ A. Tafzi, ${ }^{1}$ S. W. Chou, ${ }^{5}$ L. Veisz, ${ }^{5}$ and V. Malka ${ }^{1}$ \\ ${ }^{1}$ Laboratoire d'Optique Appliquée, ENSTA ParisTech - CNRS UMR7639 - École Polytechnique, Chemin de la Hunière, \\ 91761 Palaiseau, France \\ ${ }^{2}$ Centro de Laseres Pulsados, Parque Cientfico, 37185 Villamayor, Salamanca, Spain \\ ${ }^{3}$ Institute of Physics ASCR, v.v.i. (FZU), ELI Beamlines project, Na Slovance 2, 18221 Prague, Czech Republic \\ ${ }^{4}$ Czech Technical University in Prague, FNSPE, Brehova 7, 11519 Prague, Czech Republic \\ ${ }^{5}$ Max-Planck-Institut für Quantenoptik, Hans-Kopfermann-Strasse 1, 85748 Garching, Germany
}

(Received 8 March 2015; published 7 October 2015)

\begin{abstract}
An important limit for energy gain in laser-plasma wakefield accelerators is the dephasing length, after which the electron beam reaches the decelerating region of the wakefield and starts to decelerate. Here, we propose to manipulate the phase of the electron beam in the wakefield, in order to bring the beam back into the accelerating region, hence increasing the final beam energy. This rephasing is operated by placing an upward density step in the beam path. In a first experiment, we demonstrate the principle of this technique using a large energy spread electron beam. Then, we show that it can be used to increase the energy of monoenergetic electron beams by more than $50 \%$.
\end{abstract}

DOI: 10.1103/PhysRevLett.115.155002

PACS numbers: 52.38.Kd, 41.75.Jv, 52.38.Ph

Laser-wakefield accelerators allow the production of relativistic electron beams over a short acceleration distance (millimeter to centimeter scale) by focusing a highintensity laser pulse in an underdense plasma [1-3]. The maximum attainable energy is limited by three processes: laser pulse depletion, laser defocusing, and dephasing. Each of theses processes occurs after a characteristic propagation length and the final electron energy is determined by the process that sets in first. First, the depletion length is the distance over which the laser pulse transfers most of its energy to the wakefield and subsequently cannot sustain the wakefield any further. Increasing the energy transfer in a depletion-limited accelerator would require increasing the laser energy $[4,5]$. Second, diffraction of the laser during propagation will reduce the intensity. This effect is generally mitigated by self-focusing. However, self-focusing is not efficient over an arbitrarily long distance because the laser power decreases during the propagation, due to pump depletion, eventually becoming smaller than the critical power for self-focusing. Therefore, accelerating the electron beam over long lengths requires plasma waveguides [6,7]. Pump depletion and defocusing determine the distance over which the wakefield structure can be maintained. Yet, the excitation of a wakefield is not sufficient to guarantee that the electron beam is accelerated, because of dephasing. Actually, as the laser group velocity and thus the wake velocity are smaller than the electron beam velocity, the electron beam outruns the plasma wave during the acceleration and reaches a phase of the wake where the field is decelerating. This effect is an important limiting factor in a considerable range of experimental conditions.
The laser group velocity and hence the dephasing length depend on the plasma density, getting longer for low densities. It was proposed years ago to use a spatially tapered plasma density profile to increase this limit and overcome electron dephasing [8]. The idea behind this method is to use an accelerating medium with an upward density ramp along the laser propagation. As the driving laser pulse encounters a higher plasma density, the wakefield period shrinks and the frontier between the accelerating and decelerating region moves as fast as the electron bunch itself, keeping it at the same phase inside the ion cavity. The phase matching between the wakefield and the electron bunch can be kept for a longer acceleration distance, therefore leading to higher electron energies. To get perfect matching the density profile must be parabolic, the experimental realization of which is not straightforward. The density tapering effect has been extensively investigated numerically [9-13]; however, it has been sparsely studied experimentally as of yet [14].

In this Letter, we explore a simple way to manipulate the electron beam and increase the electron energy, with a plasma presenting a low density region followed by a high density one, separated by a sharp density jump. Ideally, the density step is placed close to the dephasing length, where the head of the bunch enters the decelerating region. When the laser crosses the density jump, the bubble shrinks abruptly [Fig. 1(c)]. Without the density step, the most energetic electrons at the head of the bunch would eventually enter the decelerating zone and their energy would decrease. In contrast, with the density step, electrons exit the decelerating region and shift almost instantly to the rear of the cavity where the accelerating field is larger, as 


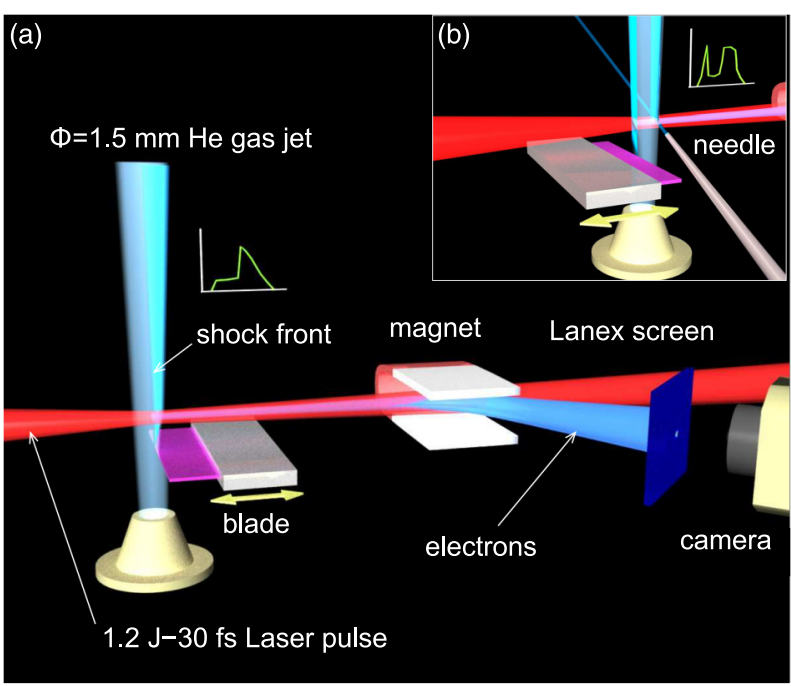

(c) Before the density step

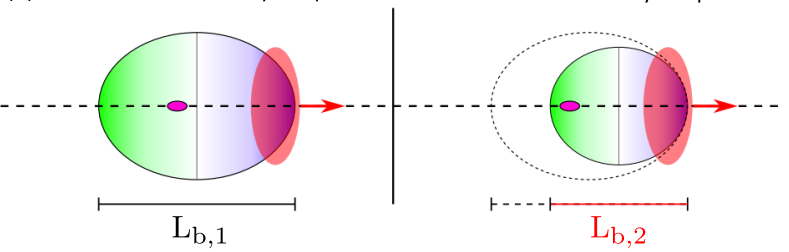

FIG. 1 (color online). Schematic representation of the first (a) and second (b) experimental setup. The blade can move in and out the gas jet. The density profile is shown in green in the two cases near the gas jet. (c) Schematic representation of the bubble before and after the density step. The driving pulse (red) generates a bubble with a size $L_{b, 1}$, which shrinks $\left(L_{b, 2}<L_{b, 1}\right)$ by crossing the density step. The accelerating (green gradient) and decelerating (blue gradient) regions are shown. The electron bunch (purple) reaches the end of the accelerating region before the density step and is shifted back to the accelerating field when crossing the density step.

shown in Fig. 1(c). The maximum electron energy is therefore larger than in the case without the transition. In a first experiment, a large energy spread electron beam is used to demonstrate the principle of this technique. The density profile is obtained by creating a shock front in a supersonic gas jet, generated by placing a blade perpendicular to the gas flow emanating from the nozzle. In a second experiment, the density step is made with a second gas jet, which can be used to enhance the energy of monoenergetic electron beams.

The experiment has been performed with the "Salle Jaune" Ti:Sa laser system (laser wavelength $\lambda_{0}=813 \mathrm{~nm}$ ) at Laboratoire d'Optique Appliquée. A linearly polarized, $1.2 \mathrm{~J}$ on target, $30 \mathrm{fs}$ (corresponding to a peak power $P=40 \mathrm{TW}$ ) laser pulse is focused at the entrance of a $1.5 \mathrm{~mm}$ supersonic helium gas jet using an $\mathrm{f} / 10$ off-axis parabola [as seen in the experimental setup sketched in Fig. 1(a)]. The full width at half maximum (FWHM) focal spot size is $18 \mu \mathrm{m}$, with a peak intensity on target of $I=1 \times 10^{19} \mathrm{~W} \cdot \mathrm{cm}^{-2}$, equivalent to a normalized vector potential $a_{0}=2.2$. A $500 \mu \mathrm{m}$ thick silicon wafer is placed on the leaving side of the gas jet to create a sharp density transition, by using a setup similar to the one in Refs. $[15,16]$. Note that in these previous studies the shock front is created on the entering side of the gas jet to trigger electron injection in the downward density jump, whereas for now the shock is on the leaving side of the jet and it creates a sharp upward density ramp. Measured longitudinal plasma density profiles for different positions of the blade in the jet are presented in the Supplemental Material [17]. The longitudinal position of the shock is adjusted by moving the blade in and out. Electron spectra are measured with a spectrometer consisting of a permanent magnet (1.1 T with a length of $100 \mathrm{~mm}$ ) combined with a phosphor screen imaged on a 16 bit CCD camera. The phosphor screen and detection system are calibrated so that the electron beam charge and energy distribution are measured for each shot.

First, a scan of the gas density is performed in order to determine the optimum plasma density for which the electron energy cutoff is the highest. The energy spectrum with a plasma density without the transition is shown in the top panel of Fig. 2(a) (angle resolved spectrum) and in red in Fig. 2(b) (spectrum integrated over the transverse direction). The electron energy distribution corresponds to the force laser wakefield regime [18], with a long plateau feature and a Maxwellian decrease with a cutoff energy around $230 \mathrm{MeV}$. The cutoff energy is defined as the electron energy where the charge of the beam becomes smaller than $18 \mathrm{fC} / \mathrm{MeV}$. Such a spectrum indicates the transverse self-injection of a long bunch [19], which is consistent with an electron plasma peak density $n_{e}=8.5 \times$ $10^{18} \mathrm{~cm}^{-3}$ along a few millimeters.

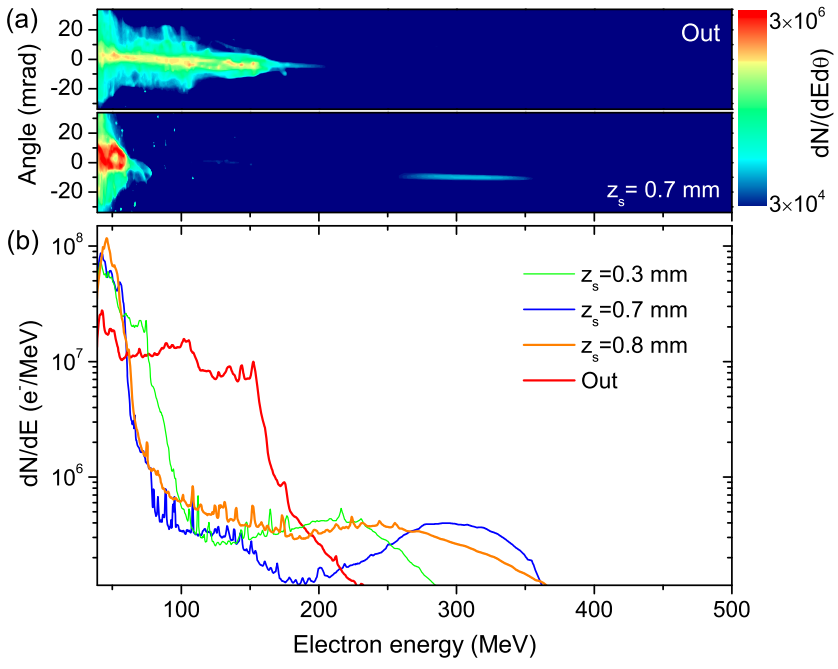

FIG. 2 (color online). (a) Experimental angle resolved electron spectra in logarithmic scale without (top panel) and with the shock at $0.7 \mathrm{~mm}$ after the gas jet center (bottom panel). (b) Angle integrated electron spectra in logarithmic scale for four positions of the blade. 
When the blade is placed such that the shock is created slightly beyond the center of the gas jet, the spectrum changes drastically, as shown in the bottom panel of Fig. 2(a) (corresponding to a shock position of $z_{s}=0.7 \mathrm{~mm}$, measured from the center of the gas nozzle at $z=0 \mathrm{~mm}$ ). Figure 2(b) shows the integrated spectrum for this shock position in blue. The number of electrons between 100 and $200 \mathrm{MeV}$ substantially drops by a factor 20, and a quasimonoenergetic peak appears around $300 \mathrm{MeV}$, with an energy-spread FWHM around 30\%. The cutoff energy at $18 \mathrm{fC} / \mathrm{MeV}$ is around $100 \mathrm{MeV}$ higher (up to $360 \mathrm{MeV}$ ) than with the flat density profile. The high energy peak, containing about $7 \mathrm{pC}$, is well collimated (divergence lower than $4 \mathrm{mrad}$ FWHM), whereas the low energy branch of the spectrum (between 50 and $100 \mathrm{MeV}$ ) presents a larger divergence (about 15 mrad FWHM) than for the case without the shock (5 mrad FWHM). Moreover, the number of low energy electrons-with energies lower than $70 \mathrm{MeV}$-is larger for the density step profile. Note that the total charge without $(Q=181 \pm 20 \mathrm{pC})$ and with $(Q=211 \pm 12 \mathrm{pC})$ the shock is similar. The energy gain of the electron bunch is easily tunable by moving the blade in the gas jet. The cutoff energy decreases when the blade moves too much into the jet or too far away from the nozzle center (see also the Supplemental Material [17]). When the shock is placed close to the dephasing length, the energy gain is optimum. However, the high energy part of the beam does not present a clear peak feature for all shock positions, as shown in Fig. 2(b).

To get insight on the details of the rephasing process, we perform simulations of the injection and acceleration of electrons along the gas jet by using the particle-in-cell code CalderCirc [20]. This fully electromagnetic 3D code uses cylindrical coordinates $(r, z)$ and Fourier decomposition in the poloidal direction. The simulations are performed using a mesh with $\Delta x=0.3 k_{0}^{-1}$ and $\Delta r=1.5 k_{0}^{-1}$ (with $\left.k_{0}=1 / \lambda_{0}\right)$, and two Fourier modes ( $m=0$ and 1 ). The plasma density profile is defined from the experimentally measured profiles, with a peak density $n_{e}=8.5 \times$ $10^{18} \mathrm{~cm}^{-3}$ for the plasma without the density transition. The laser intensity is set to $I=1.0 \times 10^{19} \mathrm{~W} \cdot \mathrm{cm}^{-2}$ and the laser waist to $15 \mu \mathrm{m}$.

Figure 3 shows a snapshot of the electron density distribution in the longitudinal phase space $(z, E)$ resulting from simulations, for a gas jet without (a) and with (b) the shock. The simulated energy spectra of the extracted electrons are shown in Fig. 3(c), presenting a distribution similar to those obtained in the experiment. Self-injection of electrons into the bubble begins relatively late during the pulse propagation, around the middle of the gas jet (at $z=0 \mathrm{~mm}$ ). For the case without the shock, self-injection of electrons will continue up to $z \sim 1.3 \mathrm{~mm}$. Accordingly, this lengthy self-injection process results in a long electron bunch, as shown in Fig. 3(a). In the case without the shock,
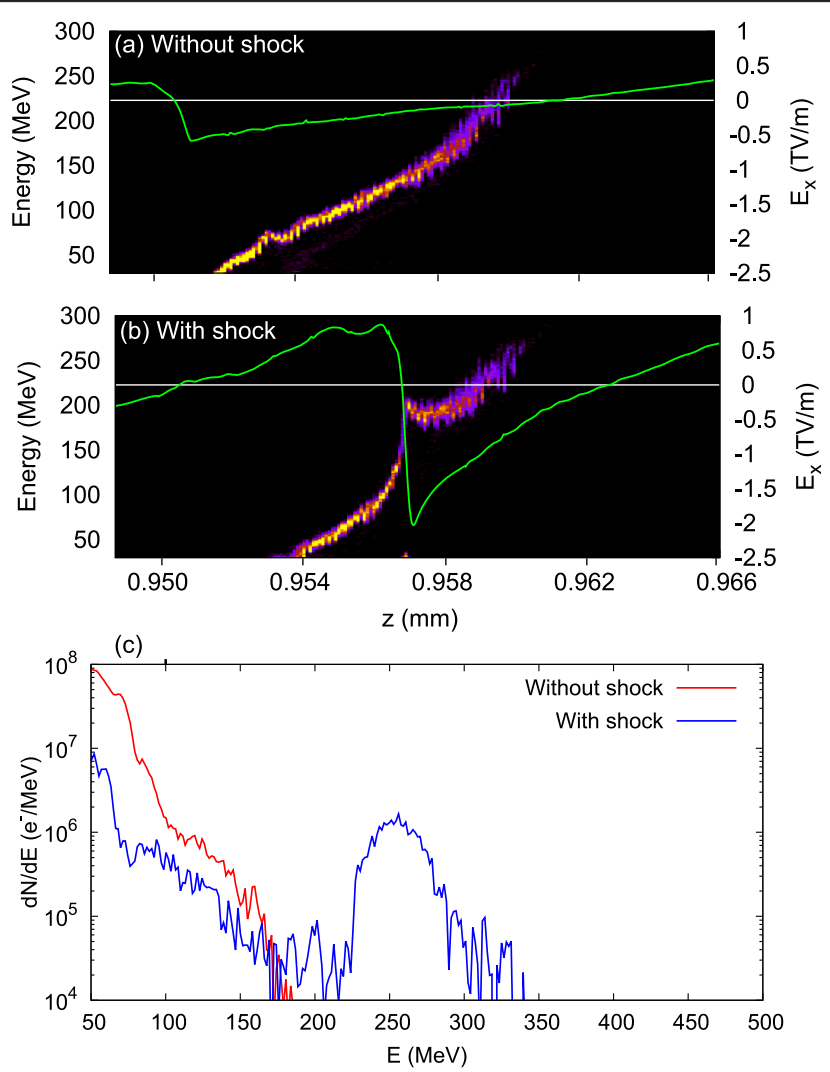

FIG. 3 (color online). Electron density in the phase space $(z, E)$ for a gas jet without (a) and with (b) the shock. The green curve shows the longitudinal electric field on the laser axis. Simulated spectra obtained at the exit are shown in (c). The density transition is at $z=0.900 \mathrm{~mm}$.

the head of the bunch reaches the decelerating region of the bubble after $\sim 0.9 \mathrm{~mm}$ of acceleration with an energy around $250 \mathrm{MeV}$ (the limit between the accelerating and decelerating regions is the point where the longitudinal field sign switches). As a result, electrons at the head of the bunch dephase and at the end of the gas jet their energy has decreased below $200 \mathrm{MeV}$, as shown in Fig. 3(c).

In the shock case, the phase space drastically changes as soon as the bunch crosses the density jump. The left side of the snapshot shown in Fig. 3(b) is about $50 \mu \mathrm{m}$ from the shock rising edge $(z=0.9 \mathrm{~mm})$. Because of the reduction of the bubble size and the resulting positive shift of the null field point, when the wakefield crosses the sharp density transition, the head of the bunch shifts back to the accelerating region. After the density step, it is located at the tail of the contracted bubble, and is efficiently accelerated by the extremely large longitudinal field of $E_{x} \approx 2 \mathrm{TV} \cdot \mathrm{m}^{-1}$. The result is a very fast rotation of the head of the bunch in the phase space $(z, E)$, which produces naturally a quasimonoenergetic spectrum, as can be seen in Fig. 3(c). Beyond the shock, the back half of the bunch is in the region with the decelerating field. It experiences also a strong defocusing field when crossing the rear of the 


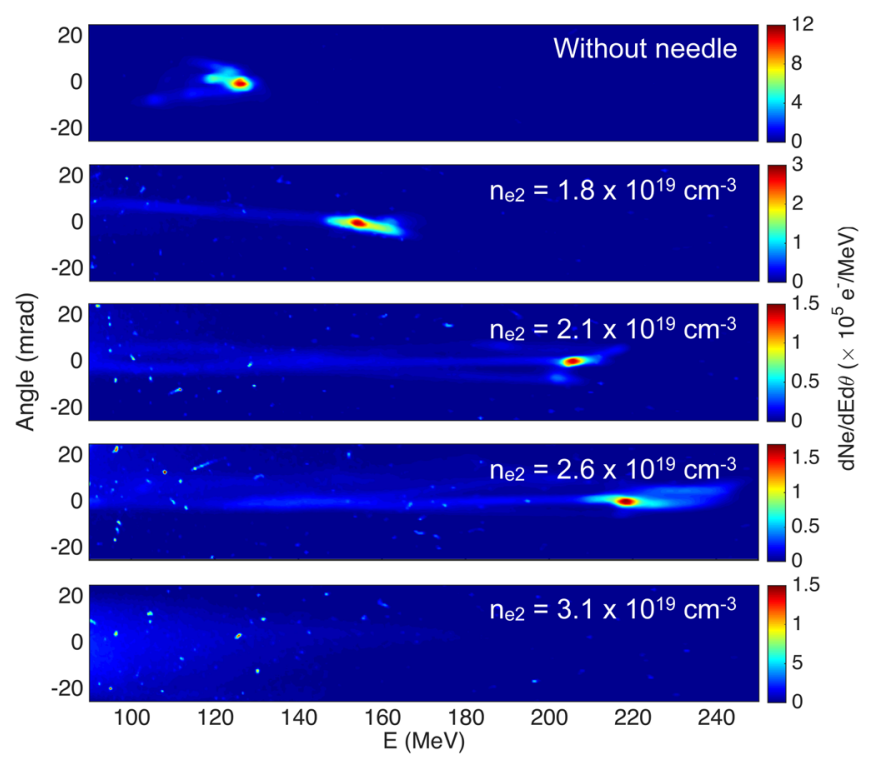

FIG. 4 (color online). Experimental angle resolved electron spectra of shock-injected electrons with no gas flowing from the needle (top panel), and for four different plasma densities in the second jet.

bubble. The corresponding electrons, in the range between 100 and $200 \mathrm{MeV}$, are both decelerated and defocused, resulting in a divergence growth at low energy. In a few simulations, it was observed that some electrons can exit the wakefield if the electrons are too strongly defocused. Note that the down-ramp density gradient after the transition can also lead in some cases to electron injection; however, their energy remains below $90 \mathrm{MeV}$ (see also the Supplemental Material [17]).

While, in Fig. 2, only the head of a large energy spread bunch is being rephased and further accelerated, this technique can also be used to rephase and reaccelerate a higher quality, low energy spread electron beam. Such an electron beam can be generated by using shock front injection $[15,16]$. The experimental setup is modified as sketched in Fig. 1(b). The silicon wafer is placed at the entrance of the jet to generate a sharp downward density gradient allowing for shock front injection of electrons. A second gas jet formed with a $500 \mu \mathrm{m}$ diameter needle is placed horizontally at the output of the first supersonic jet, creating a region with a higher tunable density $n_{e, \text { needle }}$ Because of the formation of a shock, the sharpness of the transition between these two density regions is similar to that obtained with the previous setup. The resulting plasma density profile is shown in green in Fig. 1(b).

Shock-injected electrons have a quasimonoenergetic distribution, as shown in Fig. 4(a), with a peak energy of $125 \pm 2 \mathrm{MeV}$ and a charge of $17 \pm 2 \mathrm{pC}$ (mean values over ten shots). When the needle is placed so that the transition between the two density regions is close to $z_{s}=0.55 \mathrm{~mm}$, the electron spectrum drastically changes (as shown in Fig. 4). The electron beam is rephased in the second jet after crossing the density transition. For an electron density in the second jet of $n_{e, 2}=1.8 \times 10^{19} \mathrm{~cm}^{-3}$, the peak energy increases up to $154 \pm 8 \mathrm{MeV}$. By further increasing the second jet density, electrons can reach energies of up to $220 \mathrm{MeV}$, corresponding to an energy gain of 76\%, as seen previously in Fig. 4. When the plasma density in the second density region is too high $\left(n_{e, 2}=3.1 \times 10^{19} \mathrm{~cm}^{-3}\right)$, the bubble contracts too strongly and the electron beam is located behind the cavity, and is thus decelerated and defocused.

In conclusion, an experimental demonstration of a simple density tailored wakefield accelerator was presented. More precisely, a sharp upward density gradient was used to rephase the electron beam with the accelerating field and increase its energy. This technique can either be used to select a part of a broad energy spread electron beam and increase its energy or to enhance the energy of a monoenergetic electron beam, preserving its energy spread. Experimental results highlight in both cases a maximum energy enhancement of about $50 \%$ compared with a transition-free plasma density.

This work was supported by the European Research Council through the X-Five ERC project (Contract No. 339128), LA3NET (GA-ITN-2011-289191), EuCARD2/ANAC2 EC FP7 (Contract No. 312453), DFG Project Transregio TR18, and the Association EURATOM Max-Planck-Institut fuer Plasmaphysik, and by the Agence Nationale pour la Recherche through the projects ANR-10-EQPX-CILEX and FENICS ANR-12JS04-0004-01.

[1] J. Faure, Y. Glinec, A. Pukhov, S. Kiselev, S. Gordienko, E. Lefebvre, J. P. Rousseau, F. Burgy, and V. Malka, Nature (London) 431, 541 (2004).

[2] S. P. D. Mangles, C. D. Murphy, Z. Najmudin, A. G. R. Thomas, J. L. Collier, A. E. Dangor, E. J. Divall, P. S. Foster, J. G. Gallacher, C. J. Hooker, D. A. Jaroszynski, A. J. Langley, W. B. Mori, P. A. Norreys, F. S. Tsung, R. Viskup, B. R. Walton, and K. Krushelnick, Nature (London) 431, 535 (2004).

[3] C. G. R. Geddes, C. Toth, J. van Tilborg, E. Esarey, C. B. Schroeder, D. Bruhwiler, C. Nieter, J. Cary, and W. P. Leemans, Nature (London) 431, 538 (2004).

[4] X. Wang et al., Nat. Commun. 4, 1988 (2013).

[5] H. T. Kim, K. H. Pae, H. J. Cha, I. J. Kim, T. J. Yu, J. H. Sung, S. K. Lee, T. M. Jeong, and J. Lee, Phys. Rev. Lett. 111, 165002 (2013).

[6] W. P. Leemans, B. Nagler, A. J. Gonsalves, C. Toth, K. Nakamura, C. G. R. Geddes, E. Esarey, C. B. Schroeder, and S. M. Hooker, Nat. Phys. 2, 696 (2006).

[7] W. Leemans, A. Gonsalves, H.-S. Mao, K. Nakamura, C. Benedetti, C. Schroeder, C. Tóth, J. Daniels, D. Mittelberger, S. Bulanov, J.-L. Vay, C. Geddes, and E. Esarey, Phys. Rev. Lett. 113, 245002 (2014). 
[8] P. Sprangle, B. Hafizi, J. Peñano, R. Hubbard, A. Ting, C. Moore, D. Gordon, A. Zigler, D. Kaganovich, and T. Antonsen, Phys. Rev. E 63, 056405 (2001).

[9] H. Suk, C. Kim, G. Kim, J. Kim, I. Ko, and H. Lee, Phys. Lett. A 316, 233 (2003).

[10] P. Sprangle, J. R. Peñano, B. Hafizi, R. F. Hubbard, A. Ting, D. F. Gordon, A. Zigler, and T. M. Antonsen, Phys. Plasmas 9, 2364 (2002).

[11] J. U. Kim, N. Hafz, and H. Suk, Phys. Rev. E 69, 026409 (2004).

[12] W. Rittershofer, C. B. Schroeder, E. Esarey, F. J. Grner, and W. P. Leemans, Phys. Plasmas 17, 063104 (2010).

[13] M. S. Hur and H. Suk, Phys. Plasmas 18, 033102 (2011).

[14] M. S. Kim, D. G. Jang, T. H. Lee, I. H. Nam, I. W. Lee, and H. Suk, Appl. Phys. Lett. 102, 204103 (2013).

[15] K. Schmid, A. Buck, C. M. S. Sears, J. M. Mikhailova, R. Tautz, D. Herrmann, M. Geissler, F. Krausz, and L. Veisz, Phys. Rev. ST Accel. Beams 13, 091301 (2010).
[16] A. Buck, J. Wenz, J. Xu, K. Khrennikov, K. Schmid, M. Heigoldt, J. M. Mikhailova, M. Geissler, B. Shen, F. Krausz, S. Karsch, and L. Veisz, Phys. Rev. Lett. 110, 185006 (2013).

[17] See Supplemental Material, at http://link.aps.org/ supplemental/10.1103/PhysRevLett.115.155002 for a brief description of plasma density measurement with a Nomarski interferometer and for more information on the influence of the shock position on electron energy and on the electron phase-space evolution at the density transition.

[18] V. Malka, S. Fritzler, E. Lefebvre, M. M. Aleonard, F. Burgy, J. P. Chambaret, J. F. Chemin, K. Krushelnick, G. Malka, S. P. D. Mangles, Z. Najmudin, M. Pittman, J. P. Rousseau, J. N. Scheurer, B. Walton, and A. E. Dangor, Science 298, 1596 (2002).

[19] S. Corde, C. Thaury, A. Lifschitz, G. Lambert, K. Ta Phuoc, X. Davoine, R. Lehe, D. Douillet, A. Rousse, and V. Malka, Nat. Commun. 4, 1501 (2013).

[20] A. F. Lifschitz, X. Davoine, E. Lefebvre, J. Faure, C. Rechatin, and V. Malka, J. Comput. Phys. 228, 1803 (2009). 